\title{
A Modified Homotopy Perturbation Transform Method for Transient Flow of a Third Grade Fluid in a Channel with Oscillating Motion on the Upper Wall
}

\author{
Mohammed Abdulhameed, Rozaini Roslan, and Mahathir Bin Mohamad \\ Centre for Research in Computational Mathematics, Faculty of Science, Technology and Human Development, Universiti Tun Hussein \\ Onn, 86400 Batu Pahat, Johor, Malaysia
}

Correspondence should be addressed to Mohammed Abdulhameed; muallahyidi@yahoo.com

Received 20 January 2014; Revised 19 June 2014; Accepted 19 June 2014; Published 17 July 2014

Academic Editor: Clement Kleinstreuer

Copyright (c) 2014 Mohammed Abdulhameed et al. This is an open access article distributed under the Creative Commons Attribution License, which permits unrestricted use, distribution, and reproduction in any medium, provided the original work is properly cited.

\begin{abstract}
A new analytical algorithm based on modified homotopy perturbation transform method is employed for solving the transient flow of third grade fluid in a porous channel generated by an oscillating upper wall. This method incorporates the He's polynomial into the HPM, combined with Laplace transform. Comparison with HPM and OHAM analytical solutions reveals that the proposed algorithm is highly accurate. This proves the validity and great potential of the proposed algorithm as a new kind of powerful analytical tool for transient nonlinear problems. Graphs representing the solutions are discussed, and appropriate conclusions are drawn.
\end{abstract}

\section{Introduction}

The equations describing the motion of non-Newtonian fluids are strongly of nonlinear higher order than the NavierStokes equation for Newtonian fluids. These nonlinear equations form a very complex structure, with a small number of exact solutions. Mostly, numerical methods have largely been used to handle these equations. The class of problems with known exact solution is related to the problem for infinite flat plate. The related studies in the recent years are as follows: Fakhar et al. [1] examine the exact unsteady flow of an incompressible third grade fluid along an infinite plane porous plate. They obtained results by applying a translational type of symmetries combined with finite difference method. Danish and Kumar [2] analysed a steady flow of a third grade between two parallel plates using similarity transformation. Abdulhameed et al. [3] consider an unsteady viscoelastic fluid of second grade for an infinite plate. They applied Laplace transform together with the regular perturbation techniques to obtain the exact solution. Ayub et al. [4] analysed the problem of steady flow of a third grade fluid for an infinite plate porous plate using homotopy analysis method (HAM).
Homotopy perturbation method developed by He [5] for solving linear and nonlinear initial-boundary value problem merges two techniques, the perturbation and standard homotopy. Recently, the homotopy perturbation method has been modified by some scientists to obtain more accurate results and rapid convergence and also to reduce the amount of computation. Ghorbani [6] introduced He's polynomials based on homotopy perturbation method for nonlinear differential equations. The homotopy perturbation transform method (HPTM) introduced by Khan and $\mathrm{Wu}$ [7] is a combination of the homotopy perturbation method and Laplace transform method that is used to solve various types of linear and nonlinear systems of partial differential equations. The modified homotopy perturbation transform method (MHPTM) by Khan and Smarda [8] is based on the application of Laplace transform to solve the thirdorder boundary layer equation on semi-infinite domain. Nazari-Golshan et al. [9] developed a modified homotopy perturbation Fourier transform method for nonlinear and singular Lane-Emden equations.

The goal of the present work is to present an algorithm base on MHPTM to handle the problem of transient flow 
of third grade fluid in a channel with oscillating upper wall, study the fluid behaviour in particular, and examine the effect of viscoelastic parameter on velocity field.

\section{New Analytical Algorithm}

Consider the following differential equation:

$$
F(u(t, z))=0, \quad t \geq 0, z \geq 0 .
$$

Usually the operators $F$ can be decomposed into two parts, a linear part $R$ and a nonlinear part $N$ :

$$
R(u(t, z))+N(u(t, z))=g(z) .
$$

We construct a homotopy as follows:

$$
R(u(t, z))+p N(u(t, z))=g(z),
$$

where $p \in[0,1]$ is an embedding parameter. Taking the Laplace transform of both sides of (2) we obtain

$$
L\{R(u(t, z))\}+L\{N(u(t, z))\}=L(g(z)) .
$$

Considering the linear operator $R$ in (3), the concept of the homotopy perturbation method with embedding parameter $p$ is used to generate a series expansion for $R$ as follows:

$$
R(u(t, z))=R\left(\sum_{i=0}^{\infty} p^{i} v_{i}\right)
$$

and for the nonlinear operator $N$ in (3), follow the concept of He's polynomial, $H_{n}$, as follows:

$$
N(u(t, z))=\sum_{n=0}^{\infty} p^{n} H_{n}
$$

where He's polynomials (Ghorbani [6]), $H_{n}$, are defined as

$$
H_{n}=\frac{1}{n !} \frac{d^{n}}{d p^{n}} N\left(\sum_{i=0}^{n} p^{i} u_{i}\right)_{p=0} .
$$

Substituting (5) and (6) into (4) we obtain

$$
L\left\{R\left(\sum_{i=0}^{\infty} p^{i} v_{i}\right)\right\}+L\left\{\sum_{i=0}^{\infty} p^{i+1} H_{i}\right\}=L(g(z)) .
$$

The first few components of He's polynomials, for example, are given by

$$
\begin{aligned}
& H_{0}(u)=N\left(u_{0}\right), \\
& H_{1}(u)=\frac{d}{d p} N\left(\sum_{i=0}^{1} p^{i} u_{i}\right)_{p=0}, \\
& H_{2}(u)=\frac{1}{2 !} \frac{d^{2}}{d p^{2}} N\left(\sum_{i=0}^{2} p^{i} u_{i}\right)_{p=0}, \\
& H_{3}(u)=\frac{1}{3 !} \frac{d^{3}}{d p^{3}} N\left(\sum_{i=0}^{3} p^{i} u_{i}\right)_{p=0} .
\end{aligned}
$$

However, (8) can be rewritten in the form

$$
\sum_{i=0}^{\infty} p^{i} L\left\{R\left(v_{i}\right)\right\}+\sum_{i=0}^{\infty} p^{i+1} L\left\{H_{i}\right\}=L\{g\} .
$$

Using (10) we introduce the recursive relation

$$
L\left\{R\left(v_{0}\right)\right\}=L\{g\}
$$

such that

$$
\sum_{i=1}^{\infty} p^{i} L\left\{R\left(v_{i}\right)\right\}+\sum_{i=0}^{\infty} p^{i+1} L\left\{H_{i}\right\}=0
$$

The recursive equation deduced from (12) can be rewritten as

$$
\begin{gathered}
p^{0}: L\left\{R\left(v_{0}\right)\right\}=L\{g\}, \\
p^{1}: L\left\{R\left(v_{1}\right)\right\}+L\left\{H_{0}\right\}=0, \\
p^{2}: L\left\{R\left(v_{2}\right)\right\}+L\left\{H_{1}\right\}=0, \\
p^{3}: L\left\{R\left(v_{3}\right)\right\}+L\left\{H_{2}\right\}=0, \\
\vdots \\
p^{k}: L\left\{R\left(v_{k}\right)\right\}+L\left\{H_{k-1}\right\}=0 .
\end{gathered}
$$

\section{Model of the Problem}

Consider a third grade viscoelastic fluid which is unsteady flows between two porous infinite vertical and parallel plane walls. The distance between the walls, that is, the channel width, is $2 h$. The lower plate is stationary and the upper plate is oscillating with periodic velocity $u_{w}(t)$. The lower and the upper plates are, accordingly, located in the planes $z=-h$ and $z=+h$ of an orthogonal coordinate system with $x$-axis in the direction of flow. The $z$-axis is orthogonal to the channel walls, and the origin of the axes is such that the positions of the channel walls are $z=-h$ and $z=+h$, respectively.

The fluid velocity vector $\mathbf{V}=\left[u(t, z),-v_{w}\right]$ is assumed to be parallel to the $x$-axis, so that only the $x$-component $u$ of the velocity vector does not vanish but the transpiration cross-flow velocity $v_{w}$ remains constant, where $v_{w}<0$ is the velocity of blowing and $v_{w}>0$ is the velocity of suction. Initially, both the channel walls and the fluid are at rest. The external pressure gradient is zero and the fluid velocity $\mathbf{u}(z, t)=u(z, t) \mathbf{i}$ is described by the governing equation:

$$
\begin{aligned}
\mu \frac{\partial^{2} u}{\partial z^{2}} & +\alpha_{1} \frac{\partial^{3} u}{\partial z^{2} \partial t}-\alpha_{1} v_{w} \frac{\partial^{3} u}{\partial z^{3}}+6 \beta_{3}\left(\frac{\partial u}{\partial z}\right)^{2} \frac{\partial^{2} u}{\partial z^{2}} \\
& -\rho\left(\frac{\partial u}{\partial t}-v_{w} \frac{\partial u}{\partial z}\right)=0
\end{aligned}
$$

where $\rho$ is the fluid density, $\mu$ is the coefficient of viscosity, $\alpha_{1}$ is the viscoelastic parameter for a second grade fluid, and $\beta_{3}$ is the viscoelastic parameter for a third grade fluid. 
The initial and boundary conditions are

$$
\begin{aligned}
u(0, z) & =0, \quad z \geq 0, \\
u(t,-h) & =0, \quad t>0, \\
u_{w}(t) & =u(t,+h)=u_{0} \exp (i \omega t), \quad t>0,
\end{aligned}
$$

where $u_{0}$ is the amplitude of wall oscillations, $\omega>0$ is the frequency of the wall velocity, and $i$ is the imaginary unit. Using the wall velocity $u_{w}(t)$ given in the expression (17), the cosine and sine oscillations can be obtained by taking the real and imaginary parts of the velocity field $u(t, z)$.

Consider the following set of nondimensional variables:

$$
\begin{array}{ll}
z^{*}=\frac{u_{0}}{v h} z, & u^{*}=\frac{u}{u_{0}}, \quad t^{*}=\frac{u_{0}^{2} t}{v}, \quad \omega^{*}=\frac{\omega \nu}{u_{0}^{2}}, \\
\xi^{*}=\frac{b v_{w}}{u_{0}}, & \beta^{*}=\frac{6 \beta_{3}}{\mu}\left(\frac{u_{0}}{h}\right)^{2}, \quad \alpha^{*}=\frac{\alpha_{1}}{\rho}\left(\frac{u_{0}}{h}\right)^{2} .
\end{array}
$$

We obtain the nondimensional initial-boundary values problem (dropping the $*$ notation)

$$
\begin{gathered}
\frac{\partial^{2} u}{\partial z^{2}}+\alpha \frac{\partial^{3} u}{\partial z^{2} \partial t}-\alpha \xi \frac{\partial^{3} u}{\partial z^{3}}+\beta\left(\frac{\partial u}{\partial z}\right)^{2} \frac{\partial^{2} u}{\partial z^{2}}+\xi \frac{\partial u}{\partial z}-\frac{\partial u}{\partial t}=0 \\
u(0, z)=0, \quad z \geq 0 \\
u(t,-1)=0, \quad t>0 \\
u_{w}(t)=u(t,+1)=\exp (i \omega t), \quad t>0
\end{gathered}
$$

\section{Solution Technique}

4.1. Application of New Algorithm. To solve the problem formulated in the previous section, we apply the new algorithm formulated in Section 2.

By applying the Laplace transform with respect to time $t$ of (19)-(22) we get the following problem:

$$
\begin{gathered}
(1+\alpha s) \frac{\partial^{2} \bar{u}}{\partial z^{2}}-\alpha \xi \frac{\partial^{3} \bar{u}}{\partial z^{3}}+\beta\left(\frac{\partial \bar{u}}{\partial z}\right)^{2} \frac{\partial^{2} \bar{u}}{\partial z^{2}}+\xi \frac{\partial \bar{u}}{\partial z}-s \bar{u}=0 \\
\bar{u}(s,-1)=0 \\
\bar{u}(s,+1)=\frac{1}{s-i \omega}
\end{gathered}
$$

where $\bar{u}(s, z)=\int_{0}^{\infty} u(t, z) e^{-s t} d t$ is the Laplace transform of the function $u(t, z)$. Substituting the recursive (12) into (23) leads to the following equation:

$$
\sum_{n=0}^{\infty} p^{n}\left(\alpha s \frac{\partial^{2} \bar{u}_{n}}{\partial z^{2}}-s \bar{u}_{n}\right)=\sum_{n=0}^{\infty} p^{n+1} H_{n}(s)
$$

The recursive equation deduced from (26) can be written as follows:

$$
\begin{gathered}
p^{0}: \alpha s \frac{\partial^{2}}{\partial z^{2}} \bar{u}_{0}(s)-s \bar{u}_{0}=0, \quad \bar{u}_{0}(s,-1)=0, \\
\bar{u}_{0}(s,+1)=\frac{1}{s-i \omega}, \\
p^{1}: \alpha s \frac{\partial^{2}}{\partial z^{2}} \bar{u}_{1}(s)-s \bar{u}_{1}=H_{0}(u(s)), \quad \bar{u}_{1}(s,-1)=0, \\
\bar{u}_{1}(s,+1)=0 .
\end{gathered}
$$

The solutions of the recursive (27) can be compactly written as

$$
\begin{aligned}
& \bar{u}_{0}(s, z)=\frac{e^{(1-z) / \sqrt{\alpha}}\left(-1+e^{2(1+z) / \sqrt{\alpha}}\right)}{\left(e^{4 / \sqrt{\alpha}}-1\right)(s-i \omega)}, \\
& \bar{u}_{1}(s, z) \\
& =\frac{1}{8 s \alpha^{5 / 2}\left(e^{4 / \sqrt{\alpha}}-1\right)^{4}(s-i \omega)^{3}} \\
& \times\left\{e^{(1-3 z) / \sqrt{\alpha}}\right. \\
& \times\left[-\beta \sqrt{\alpha} e^{2 / \sqrt{\alpha}}+\beta \sqrt{\alpha} e^{6 / \sqrt{\alpha}}\right. \\
& -\beta \sqrt{\alpha} e^{6(2+z) / \sqrt{\alpha}} \\
& +\beta \sqrt{\alpha} e^{(8+6 z) / \sqrt{\alpha}}+e^{2 z / \sqrt{\alpha}} \\
& \times\left(4 s^{2}(-1+z) \alpha+\beta \sqrt{\alpha}\right. \\
& \left.+8 s i(-1+z) \alpha \omega-4(-1+z) \alpha \omega^{2}\right) \\
& +e^{2(7+2 z) / \sqrt{\alpha}}\left(-4 s^{2}(-1+z) \alpha+\beta \sqrt{\alpha}\right. \\
& +8 s i(-1+z) \alpha \omega \\
& \left.+4(-1+z) \alpha \omega^{2}\right)-3 \xi \\
& +e^{2+4 z / \sqrt{\alpha}}\left(4 s^{2}(3+z) \alpha-\beta \sqrt{\alpha}\right. \\
& \left.-8 s i(3+z) \alpha \omega-4(3+z) \alpha \omega^{2}\right) \\
& +e^{2(6+z) / \sqrt{\alpha}}\left(-4 s^{2}(3+z) \alpha-\beta \sqrt{\alpha}\right. \\
& \left.+8 s i(3+z) \alpha \omega+4(3+z) \alpha \omega^{2}\right) \\
& -4 e^{2(2+z) / \sqrt{\alpha}}\left(s^{2}(\alpha+3 z \alpha)+\beta-z \beta\right. \\
& \left.-2 s i(1+3 z) \alpha \omega-(1+3 z) \alpha \omega^{2}\right) \\
& +4 e^{2(4+z) / \sqrt{\alpha}}\left(s^{2}(5+3 z \alpha) \alpha-(3+z) \beta\right. \\
& \left.-2 s i(5+3 z) \alpha \omega-(5+3 z) \alpha \omega^{2}\right) \\
& -4 e^{6+4 z / \sqrt{\alpha}}\left(s^{2}(5+3 z \alpha) \alpha-(3+z) \beta\right. \\
& -2 s i(5+3 z) \alpha \omega \\
& \left.\left.\left.-(5+3 z) \alpha \omega^{2}\right)\right]\right\} \text {. }
\end{aligned}
$$


Using the Maple symbolic code, the inverse Laplace transform of (28) is

$$
\begin{aligned}
& u_{0}(t, z)=\frac{e^{(1-z) / \sqrt{\alpha}}\left(-1+e^{2(1+z) / \sqrt{\alpha}}\right)}{\left(e^{4 / \sqrt{\alpha}}-1\right)}\{\cos (\omega t)+i \sin (\omega t)\} \\
& u_{1}(t, z) \\
& =\frac{1}{16 \alpha^{5 / 2}\left(e^{4 / \sqrt{\alpha}}-1\right)^{4} \omega^{3}} \\
& \times\left\{e^{(1-3 z) / \sqrt{\alpha}}\left(\cos \left(\frac{\omega t}{2}\right)+i \sin \left(\frac{\omega t}{2}\right)\right)\right. \\
& \times\left[i t \left(-4 e^{2(2+z) / \sqrt{\alpha}}(-1+z)+4 e^{2(5+2 z) / \sqrt{\alpha}}\right.\right. \\
& \times(-1+z)+4 e^{2(4+z) / \sqrt{\alpha}}(3+z) \\
& -4 e^{(6+4 z) / \sqrt{\alpha}}(3+z)+\sqrt{\alpha} e^{2 / \sqrt{\alpha}}-\sqrt{\alpha} e^{6 / \sqrt{\alpha}} \\
& -\sqrt{\alpha} e^{2 z / \sqrt{\alpha}}+\sqrt{\alpha} e^{6(2+z) / \sqrt{\alpha}}+\sqrt{\alpha} e^{2(6+z) / \sqrt{\alpha}} \\
& -\sqrt{\alpha} e^{2(7+2 z) / \sqrt{\alpha}}-3 \xi \\
& \left.+\sqrt{\alpha} e^{2(2+4 z) / \sqrt{\alpha}}-\sqrt{\alpha} e^{(8+6 z) / \sqrt{\alpha}}\right) \\
& +\beta \omega(2 i+t \omega) \cos \left(\frac{\omega t}{2}\right) \\
& -\beta \sqrt{\alpha} e^{2 / \sqrt{\alpha}}\left(-4+2 i t \omega+t^{2} \omega^{2}\right) \\
& +\beta \sqrt{\alpha} e^{6 / \sqrt{2}}\left(-4+2 i t \omega+t^{2} \omega^{2}\right) \\
& -\beta \sqrt{\alpha} e^{6(2+z) / \sqrt{\alpha}}\left(-4+2 i t \omega+t^{2} \omega^{2}\right) \\
& +\beta \sqrt{\alpha} e^{(8+6 z) / \sqrt{\alpha}}\left(-4+2 i t \omega+t^{2} \omega^{2}\right) \\
& +\sqrt{\alpha} e^{(2+4 z) / \sqrt{\alpha}} \\
& \times\left(16(3+z) \sqrt{\alpha} \omega^{2}-\beta\left(-4+2 i t \omega+t^{2} \omega^{2}\right)\right) \\
& +\sqrt{\alpha} e^{2(7+2 z) / \sqrt{\alpha}} \\
& \times\left(-16(-1+z) \sqrt{\alpha} \omega^{2}+\beta\left(-4+2 i t \omega+t^{2} \omega^{2}\right)\right) \\
& +\sqrt{\alpha} e^{2 z / \sqrt{\alpha}} \\
& \times\left(16(-1+z) \sqrt{\alpha} \omega^{2}+\beta\left(-4+2 i t \omega+t^{2} \omega^{2}\right)\right) \\
& -\sqrt{\alpha} e^{2(6+z) / \sqrt{\alpha}} \\
& \times\left(16(3+z) \sqrt{\alpha} \omega^{2}+\beta\left(-4+2 i t \omega+t^{2} \omega^{2}\right)\right) \\
& +4 e^{2(2+z) / \sqrt{\alpha}} \\
& \times\left(-4(1+3 z) \alpha \omega^{2}\right.
\end{aligned}
$$

$$
\begin{aligned}
& \left.+(-1+z) \beta\left(-4+2 i t \omega+t^{2} \omega^{2}\right)\right) \\
- & 4 e^{2(5+2 z) / \sqrt{\alpha}} \\
\times & \left(-4(1+3 z) \alpha \omega^{2}\right. \\
& \left.+(-1+z) \beta\left(-4+2 i t \omega+t^{2} \omega^{2}\right)\right) \\
- & 4 e^{2(4+2 z) / \sqrt{\alpha}} \\
\times & \left(-4(5+3 z) \alpha \omega^{2}\right. \\
& \left.+(3+z) \beta\left(-4+2 i t \omega+t^{2} \omega^{2}\right)\right) \\
+ & 4 e^{(6+z) / \sqrt{\alpha}} \\
\times & \left(-4(5+3 z) \alpha \omega^{2}+(3+z)\right. \\
& \left.\left.\left.\times \beta\left(-4+2 i t \omega+t^{2} \omega^{2}\right)\right) \sin \left(\frac{\omega t}{2}\right)\right]\right\},
\end{aligned}
$$

Consequently, the first-order approximate analytical solution of (19) is given by

$$
\begin{aligned}
& u(t, z) \\
& =\frac{e^{(1-z) / \sqrt{\alpha}}\left(-1+e^{2(1+z) / \sqrt{\alpha}}\right)}{\left(e^{4 / \sqrt{\alpha}}-1\right)}\{\cos (\omega t)+i \sin (\omega t)\}, \\
& +\frac{1}{16 \alpha^{5 / 2}\left(e^{4 / \sqrt{\alpha}}-1\right)^{4} \omega^{3}} \\
& \times\left\{e^{(1-3 z) / \sqrt{\alpha}}\left(\cos \left(\frac{\omega t}{2}\right)+i \sin \left(\frac{\omega t}{2}\right)\right)\right. \\
& \times\left[i t \left(-4 e^{2(2+z) / \sqrt{\alpha}}(-1+z)\right.\right. \\
& +4 e^{2(5+2 z) / \sqrt{\alpha}}(-1+z) \\
& +4 e^{2(4+z) / \sqrt{\alpha}}(3+z) \\
& -4 e^{(6+4 z) / \sqrt{\alpha}}(3+z) \\
& +\sqrt{\alpha} e^{2 / \sqrt{\alpha}}-\sqrt{\alpha} e^{6 / \sqrt{\alpha}}-\sqrt{\alpha} e^{2 z / \sqrt{\alpha}} \\
& +\sqrt{\alpha} e^{6(2+z) / \sqrt{\alpha}}+\sqrt{\alpha} e^{2(6+z) / \sqrt{\alpha}} \\
& -\sqrt{\alpha} e^{2(7+2 z) / \sqrt{\alpha}}-3 \xi \\
& \left.+\sqrt{\alpha} e^{2(2+4 z) / \sqrt{\alpha}}-\sqrt{\alpha} e^{(8+6 z) / \sqrt{\alpha}}\right) \\
& +\beta \omega(2 i+t \omega) \cos \left(\frac{\omega t}{2}\right) \\
& -\beta \sqrt{\alpha} e^{2 / \sqrt{\alpha}}\left(-4+2 i t \omega+t^{2} \omega^{2}\right) \\
& +\beta \sqrt{\alpha} e^{6 / \sqrt{\alpha}}\left(-4+2 i t \omega+t^{2} \omega^{2}\right)
\end{aligned}
$$




$$
\begin{aligned}
& \beta \sqrt{\alpha} e^{6(2+z) / \sqrt{\alpha}}\left(-4+2 i t \omega+t^{2} \omega^{2}\right) \\
+ & \beta \sqrt{\alpha} e^{(8+6 z) / \sqrt{\alpha}}\left(-4+2 i t \omega+t^{2} \omega^{2}\right) \\
+ & \sqrt{\alpha} e^{(2+4 z) / \sqrt{\alpha}} \\
\times & \left(16(3+z) \sqrt{\alpha} \omega^{2}-\beta\left(-4+2 i t \omega+t^{2} \omega^{2}\right)\right) \\
+ & \sqrt{\alpha} e^{2(7+2 z) / \sqrt{\alpha}} \\
\times & \left(-16(-1+z) \sqrt{\alpha} \omega^{2}+\beta\left(-4+2 i t \omega+t^{2} \omega^{2}\right)\right) \\
+ & \sqrt{\alpha} e^{2 z / \sqrt{\alpha}} \\
\times & \left(16(-1+z) \sqrt{\alpha} \omega^{2}+\beta\left(-4+2 i t \omega+t^{2} \omega^{2}\right)\right) \\
& \left.\left.\left.\times \beta\left(-4+2 i t \omega+t^{2} \omega^{2}\right)\right) \sin \left(\frac{\omega t}{2}\right)\right]\right\} . \\
+ & 4 e^{(6+z) / \sqrt{\alpha}} \\
\times & (-4(5+z) / \sqrt{\alpha} \\
\times & \left(-4\left(5(3+z) \sqrt{\alpha} \omega^{2}+\beta\left(-4+2 i t \omega+t^{2} \omega^{2}\right)\right)\right. \\
& +(-1+z) \beta\left(-4 \omega^{2}\right. \\
+ & 4 e^{2(2+z) / \sqrt{\alpha}} \\
\times & \left(-4(1+3 z) \alpha \omega^{2}\right. \\
& \left.+(-1+z) \beta\left(-4+2 i t \omega+t^{2} \omega^{2}\right)\right) \\
& (-4+2 z) / \sqrt{\alpha} \\
+ &
\end{aligned}
$$

4.2. Application of Homotopy Perturbation Method (HPM). Rewrite (19) as

$$
\frac{\partial}{\partial t}\left(u-\alpha \frac{\partial^{2} u}{\partial z^{2}}\right)=\frac{\partial^{2} u}{\partial z^{2}}-\alpha \xi \frac{\partial^{3} u}{\partial z^{3}}+\beta\left(\frac{\partial u}{\partial z}\right)^{2} \frac{\partial^{2} u}{\partial z^{2}}+\xi \frac{\partial u}{\partial z} .
$$

Integrate (31) with respect tot over the interval $[0, t]$ as follows:

$$
u-\alpha \frac{\partial^{2} u}{\partial z^{2}}=\int_{0}^{t}\left[\frac{\partial^{2} u}{\partial z^{2}}-\alpha \xi \frac{\partial^{3} u}{\partial z^{3}}+\beta\left(\frac{\partial u}{\partial z}\right)^{2} \frac{\partial^{2} u}{\partial z^{2}}+\xi \frac{\partial u}{\partial z}\right] \partial t .
$$

According to the HPM technique by $\mathrm{He}$ [5], we construct the following homotopy $v(t, z ; q): R \times[0,1] \rightarrow R$ which satisfies the following relation:

$$
L(v)-L\left(u_{0}\right)+p\left[L\left(u_{0}\right)+N(v)\right]=0,
$$

where $L(v)=v-\alpha\left(\partial^{2} v / \partial z^{2}\right), N(v)=\int_{0}^{t}\left[\left(\partial^{2} v / \partial z^{2}\right)-\right.$ $\left.\alpha \xi\left(\partial^{3} v / \partial z^{3}\right)+\beta(\partial v / \partial z)^{2}\left(\partial^{2} v / \partial z^{2}\right)+\xi(\partial v / \partial z)\right] \partial t$, and $u_{0}$ is an initial approximation to the transient solution $u(t, z)$.

Taking $p$ as small parameter we assume a power series solution of (33) in the form

$$
v(t, z ; p)=\sum_{k=0}^{\infty} p^{k} v_{k}(t, z)
$$

where $v_{k}(t, z)$ are unknown functions of $t, z$. Now letting $p \rightarrow 1,(34)$ yields the approximate solution of $u(t, z)$ in the following form:

$$
u(t, z)=\lim _{q \rightarrow 1} v(t, z ; p)=\sum_{k=0}^{\infty} v_{k}(t, z) .
$$

We now substitute (34) into (33) and the initial and boundary conditions (20)-(22) and equate the coefficients of like powers of $p$ to obtain first-order problem:

$$
\begin{gathered}
p^{0}: L\left(v_{0}\right)-L\left(u_{0}\right)=0, \\
v_{0}(t,-1)=0, \quad v_{0}(t,+1)=e^{i \omega t}, \\
p^{1}: L\left(v_{1}\right)-L\left(u_{0}\right) \\
+\int_{0}^{t}\left[\frac{\partial^{2} v_{0}}{\partial z^{2}}-\alpha \xi \frac{\partial^{3} v_{0}}{\partial z^{3}}+\beta\left(\frac{\partial v_{0}}{\partial z}\right)^{2} \frac{\partial^{2} v_{0}}{\partial z^{2}}+\xi \frac{\partial v_{0}}{\partial z}\right] \partial t=0, \\
v_{1}(t,-1)=0, \quad v_{1}(t,+1)=0 .
\end{gathered}
$$

We can now solve these problems to find $v_{0}$ and $v_{1}$ :

$$
\begin{aligned}
v_{0} & =\frac{\left(e^{2(1+z) / \sqrt{\alpha}}-1\right)}{\left(e^{2(1+z) / \sqrt{\alpha}}-1\right)}\left[e^{((1-z) / \sqrt{\alpha})+i \omega t}\right] . \\
v_{1}= & \frac{1}{8 \alpha^{5 / 2}\left(e^{4 / \sqrt{\alpha}}-1\right)^{4}} \\
& \times\left\{e^{((1-3 z) / \sqrt{\alpha})+i \omega t} t\right.
\end{aligned}
$$




$$
\begin{aligned}
\times[ & -4 e^{2 z / \sqrt{\alpha}}(-1+z) \alpha+4 e^{2(7+2 z) / \sqrt{\alpha}}(-1+z) \alpha \\
& +4 e^{2(6+z) / \sqrt{\alpha}}(3+z) \alpha \\
& -4 e^{(2+4 z) / \sqrt{\alpha}}(3+z) \alpha-3 \xi+4 e^{2(2+z) / \sqrt{\alpha}} \\
& \times(1+3 z) \alpha-4 e^{2(5+2 z) / \sqrt{\alpha}}(1+3 z) \alpha \\
& -4 e^{2(4+z) / \sqrt{\alpha}}(5+3 z) \alpha \\
& +4 e^{(6+4 z) / \sqrt{\alpha}}(5+3 z) \alpha-4 e^{2(2+z+i \omega t \sqrt{\alpha}) / \sqrt{\alpha}} \\
& \times(-1+z) \beta+4 e^{2(5+2 z+i \omega t \sqrt{\alpha}) / \sqrt{\alpha}}(-1+z) \beta \\
& +4 e^{2(4+z+i \omega t \sqrt{\alpha})}(3+z) \beta \\
& -4 e^{6+4 z+2 i \omega t \sqrt{\alpha}}(3+z) \beta+e^{(2 / \sqrt{\alpha})+2 i \omega t} \sqrt{\alpha} \beta \\
& -e^{(6 / \sqrt{\alpha})+2 i \omega t} \sqrt{\alpha} \beta-e^{(2 z / \sqrt{\alpha})+2 i \omega t} \sqrt{\alpha} \beta \\
& +e^{2(6+4 z+2 i \omega t \sqrt{\alpha})} \sqrt{\alpha} \beta-e^{2(7+2 z+i \omega t \sqrt{\alpha})} \sqrt{\alpha} \beta \\
& +e^{2(6+3 z+i \omega t \sqrt{\alpha}) / \sqrt{\alpha}} \sqrt{\alpha} \beta \\
& +e^{(2+4 z+i \omega t \sqrt{\alpha}) / \sqrt{\alpha}} \sqrt{\alpha} \beta \\
& \left.\left.-e^{(8+6 z+2 i \omega t \sqrt{\alpha}) / \sqrt{\alpha}} \sqrt{\alpha} \beta\right]\right\}, \\
& (-1) \\
&
\end{aligned}
$$

The first-order approximate solution of (19) by HPM method is

$$
\begin{aligned}
u(t, z)= & v_{0}+v_{1} \\
= & \frac{\left(e^{2(1+z) / \sqrt{\alpha}}-1\right)}{\left(e^{2(1+z) / \sqrt{\alpha}}-1\right)}\left[e^{((1-z) / \sqrt{\alpha})+i \omega t}\right] \\
& +\frac{1}{8 \alpha^{5 / 2}\left(e^{4 / \sqrt{\alpha}}-1\right)} \\
& \times\left\{e^{((1-3 z) / \sqrt{\alpha})+i \omega t} t\right. \\
& \times\left[-4 e^{2 z / \sqrt{\alpha}}(-1+z) \alpha\right. \\
& +4 e^{2(7+2 z) / \sqrt{\alpha}}(-1+z) \alpha \\
& +4 e^{2(6+z) / \sqrt{\alpha}}(3+z) \alpha-4 e^{(2+4 z) / \sqrt{\alpha}} \\
& \times(3+z) \alpha-3 \xi+4 e^{2(2+z) / \sqrt{\alpha}}(1+3 z) \alpha \\
& -4 e^{2(5+2 z) / \sqrt{\alpha}}(1+3 z) \alpha \\
& -4 e^{2(4+z) / \sqrt{\alpha}}(5+3 z) \alpha
\end{aligned}
$$

$$
\begin{aligned}
& +4 e^{(6+4 z) / \sqrt{\alpha}}(5+3 z) \alpha \\
& -4 e^{2(2+z+i \omega t \sqrt{\alpha}) / \sqrt{\alpha}}(-1+z) \beta \\
& +4 e^{2(5+2 z+i \omega t \sqrt{\alpha}) / \sqrt{\alpha}}(-1+z) \beta \\
& +4 e^{2(4+z+i \omega t \sqrt{\alpha})}(3+z) \beta \\
& -4 e^{6+4 z+2 i \omega t \sqrt{\alpha}}(3+z) \beta \\
& +e^{(2 / \sqrt{\alpha})+2 i \omega t} \sqrt{\alpha} \beta-e^{(6 / \sqrt{\alpha})+2 i \omega t} \sqrt{\alpha} \beta \\
& -e^{(2 z / \sqrt{\alpha})+2 i \omega t} \sqrt{\alpha} \beta+e^{2(6+4 z+2 i \omega t \sqrt{\alpha})} \sqrt{\alpha} \beta \\
& -e^{2(7+2 z+i \omega t \sqrt{\alpha})} \sqrt{\alpha} \beta \\
& +e^{2(6+3 z+i \omega t \sqrt{\alpha}) / \sqrt{\alpha}} \sqrt{\alpha} \beta \\
& +e^{(2+4 z+i \omega t \sqrt{\alpha}) / \sqrt{\alpha}} \sqrt{\alpha} \beta \\
& \left.\left.-e^{(8+6 z+2 i \omega t \sqrt{\alpha}) / \sqrt{\alpha}} \sqrt{\alpha} \beta\right]\right\} .
\end{aligned}
$$

4.3. Application of Optimal Homotopy Asymptotic Method (OHAM). By means of the OHAM proposed by Marinca and Herişanu [10], we construct an optimal homotopy $\phi(t, z ; p): R \times[0,1] \rightarrow R$ which satisfies the following relation:

$$
\begin{aligned}
& L(\phi(t, z ; p)) \\
& \quad=H(z ; p)(L(\phi(t, z ; p))+N(\phi(t, z ; p))) .
\end{aligned}
$$

We have a great freedom to choose the auxiliary function $H(t, z ; p)$ as

$$
H(t, z ; p)=C_{1}+C_{2} e^{-z}+C_{3} t e^{-2 z},
$$

where $C_{1}, C_{2}$, and $C_{3}$ are functions depending on the variable $t, z$.

Let us consider the solutions of (39) in the form

$$
\phi\left(t, z, p ; K_{i}\right)=u_{0}(t, z)+\sum_{k=1}^{\infty} u_{k}\left(t, z ; K_{i}\right) q^{k}, \quad i=1,2, \ldots
$$

Substituting (41) into (39) and equating the coefficients of like powers of $p$ we obtain the governing equations of $u_{k}(t, z)$; that is,

$$
\begin{aligned}
& L\left(u_{k}(t, z)\right)-L\left(u_{k-1}(t, z)\right) \\
& =K_{k} N_{0}\left(u_{0}(t, z)\right) \\
& \quad+\sum_{i=1}^{k-1} K_{i}\left[L\left(u_{k-i}(t, z)\right)\right. \\
& \left.\quad+N_{(k-i)}\left(u_{0}(t, z), u_{1}(t, z), \ldots u_{k-i}(t, z)\right)\right] .
\end{aligned}
$$


The first-order approximate solution of the problem is

$$
u(t, z)=u_{0}(t, z)+u_{1}(t, z)
$$

where the zeroth-order and first-order problems from (42) are

$$
\begin{gathered}
u_{0}-\alpha \frac{\partial^{2} u_{0}}{\partial z^{2}}=0, \\
u_{0}(t,-1)=0, \quad u_{0}(t,+1)=e^{i \omega t}, \\
u_{1}-\alpha \frac{\partial^{2} u_{1}}{\partial z^{2}} \\
=\left(C_{1}+C_{2} e^{-z}+C_{3} t e^{-2 z}\right) \\
\times\left\{\int _ { 0 } ^ { t } \left[\frac{\partial^{2} u_{0}}{\partial z^{2}}-\alpha \xi \frac{\partial^{3} u_{0}}{\partial z^{3}}\right.\right. \\
\left.\left.+\beta\left(\frac{\partial u_{0}}{\partial z}\right)^{2} \frac{\partial^{2} u_{0}}{\partial z^{2}}+\xi \frac{\partial u_{0}}{\partial z}\right] \partial t\right\}, \\
u_{1}(t,-1)=0, \\
u_{1}(t,+1)=0 .
\end{gathered}
$$

The solution of (44) can be solved using widely symbolic computational Maple software. The values of the constants $C_{1}, C_{2}$, and $C_{3}$ are obtained using collocation method.

\section{Analysis of Result}

In Figure 1 and Table 1, we show that the transient approximate solutions obtained using newly technique are in better agreement with the OHAM method, as compared to the HPM solutions, for small values of time $t$ and different values of all the non-Newtonian parameters and constants. This proves that the accuracy of the solution obtained by the new method is significant and more accurate for small values of the time $t$. However, in Figure 2 and Table 2 for large values of time $t$, the transient approximate solutions obtained using newly method become divergent with the approximate solutions obtained using HPM and OHAM which proves that the accuracy of the new method fails for growing values of time $t$.

To see the physical impact of the oscillating walls on the third grade fluid on the flow field, we have plotted the graphs for velocity profiles given by (30). Figure 3 is plotted to show the variation for different amplitude of wall oscillations $\omega$ in cases of cosine and sine oscillation; it is noted that the results are physically satisfied with different values $\omega$ for both cosine and sine excitation of the upper wall. Figure 4 shows the effect of third grade viscoelastic parameter with blowing case on the velocity profile for small values of time $t$; it is clearly seen that, by increasing $\beta$, the velocity increases across the channel, and this increase is rapid near the upper wall. This abrupt change in velocity near the upper wall is due to oscillatory nature of the wall boundary which generates depressive harmonic waves into the velocity field. From this figure we can also compare the velocity field of third grade fluid with corresponding velocity field of second grade fluid

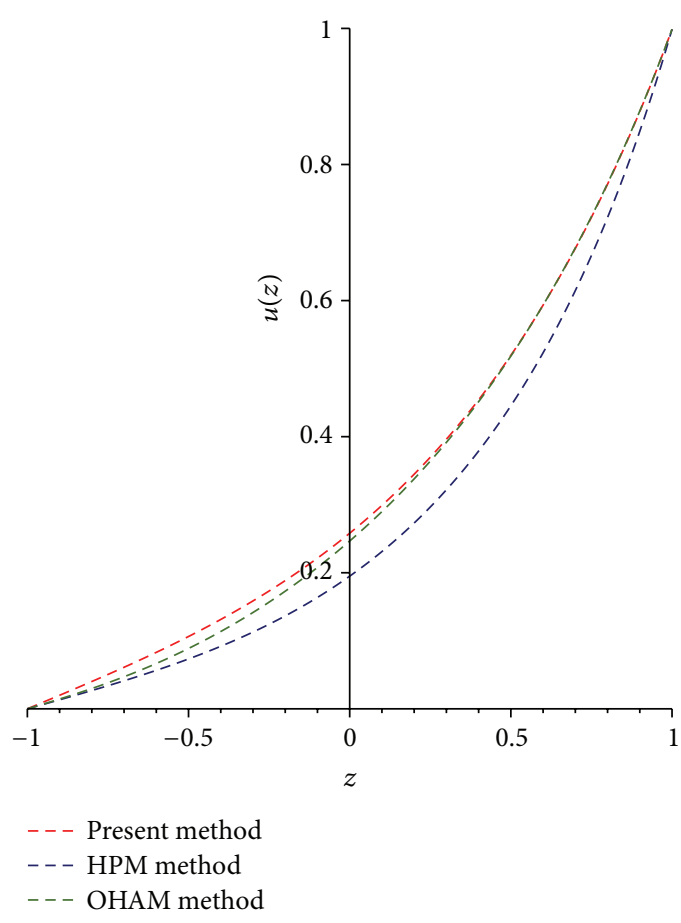

FIgURE 1: Comparison of the present results with HPM and OHAM for small value of time $t$.

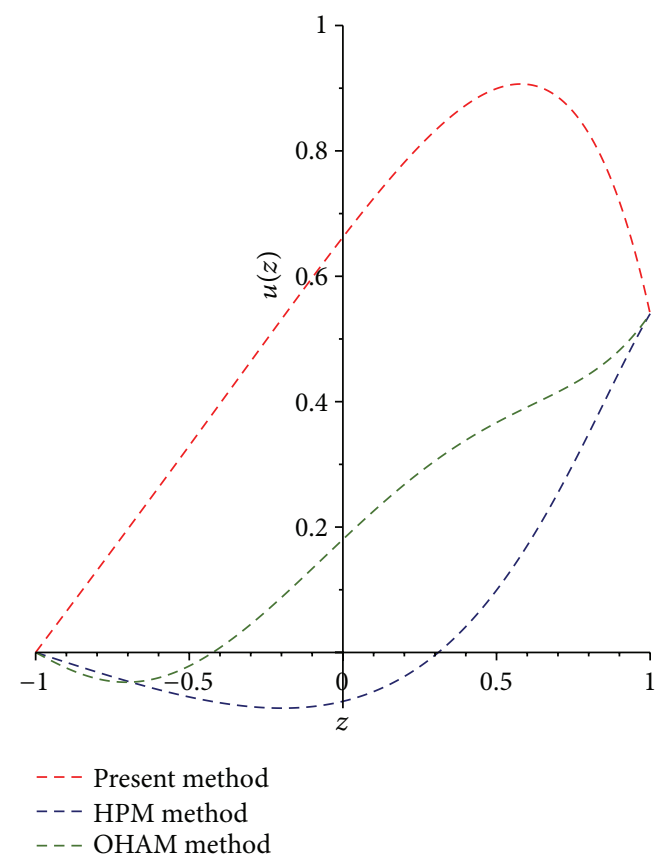

FIgURE 2: Comparison of the present results with HPM and OHAM for large value of time $t$.

$(\beta=0)$. For both cosine and sine oscillation, the third grade fluid flows faster than second grade fluid across the channel. It is noted from Figure 5 that, as we increase the value of the time $t$, the influence of the third grade parameter $\beta$ on the fluid motion is not significant as compared in Figure 4 for small values of time $t$. 
TABLE 1: Numerical results show the comparison of new algorithm results with the results obtained from HPM and OHAM for the case of small value of time $t(t=0.1)$, when $\alpha=0.5, \beta=0.5, \xi=-0.5, \omega=0.5, C_{1}=0.15419514, C_{2}=-5.54965331$, and $C_{2}=4.10010429$.

\begin{tabular}{lccccc}
\hline$z$ & New algorithm & HPM method & OHAM method & $\begin{array}{c}\text { Absolute error } \\
\text { HPM method }\end{array}$ & $\begin{array}{c}\text { Absolute error } \\
\text { OHAM method }\end{array}$ \\
\hline-1 & $-1.43639 \times 10^{-16}$ & $3.03836 \times 10^{-17}$ & $4.44089 \times 10^{-16}$ & $2.89467 \times 10^{-17}$ & $5.87728 \times 10^{-16}$ \\
-0.8 & 0.0401522 & 0.0269116 & 0.0315324 & 0.0132406 & 0.0086198 \\
-0.6 & 0.0829870 & 0.0565498 & 0.0698092 & 0.0264372 & 0.0131778 \\
-0.4 & 0.1313580 & 0.0919104 & 0.1180830 & 0.0394476 & 0.0132750 \\
-0.2 & 0.1884740 & 0.1365570 & 0.1783360 & 0.051917 & 0.0101380 \\
0 & 0.2580990 & 0.1949830 & 0.2527330 & 0.071702 & 0.0053660 \\
0.2 & 0.3447890 & 0.2730870 & 0.3443530 & 0.075333 & 0.0004360 \\
0.4 & 0.4541750 & 0.3788420 & 0.4576250 & 0.070013 & 0.0034500 \\
0.6 & 0.5933060 & 0.5232930 & 0.5986640 & 0.048897 & 0 \\
0.8 & 0.7710760 & 0.7221790 & 0.7755630 & 0.0053580 & 0 \\
1 & 0.9987500 & 0.998750 & 0.0044870 & 0 \\
\hline
\end{tabular}

TABLE 2: Numerical results show the comparison of new algorithm results with the results obtained from HPM and OHAM for the case of large value of time $t(t=2)$, when $\alpha=0.5, \beta=0.5, \xi=-0.5, \omega=0.5, C_{1}=0.15419514, C_{2}=-5.54965331$, and $C_{2}=4.10010429$.

\begin{tabular}{lccccc}
\hline$z$ & New method & HPM method & OHAM method & $\begin{array}{c}\text { Absolute error } \\
\text { HPM method }\end{array}$ & $\begin{array}{c}\text { Absolute error } \\
\text { OHAM method }\end{array}$ \\
\hline-1 & $-6.41703 \times 10^{-16}$ & $2.16947 \times 10^{-16}$ & $-1.33227 \times 10^{-15}$ & $8.5865 \times 10^{-16}$ & $6.550257 \times 10^{-16}$ \\
-0.8 & 0.1309460 & -0.0313209 & -0.0275524 & 0.1622669 & 0.1584984 \\
-0.6 & 0.2630750 & -0.0593798 & -0.0138010 & 0.3224548 & 0.2768760 \\
-0.4 & 0.3968690 & -0.0802786 & 0.0436640 & 0.4771476 & 0.3532050 \\
-0.2 & 0.5312480 & -0.0888351 & 0.1294880 & 0.7403246 & 0.4017600 \\
0 & 0.6623540 & -0.0779706 & 0.2239000 & 0.8200037 & 0.4384540 \\
0.2 & 0.7816650 & -0.0383387 & 0.3090160 & 0.8316530 & 0.4726490 \\
0.4 & 0.8728400 & 0.041187 & 0.3730220 & 0.4958180 & 0.4908880 \\
0.6 & 0.9061440 & 0.170557 & 0.4152560 & 0.4788530 & 0 \\
0.8 & 0.8279990 & 0.349146 & 0.5403020 & 0 & 0.3737000 \\
1 & 0.5403020 & 0.540302 & 0 & 0 \\
\hline
\end{tabular}

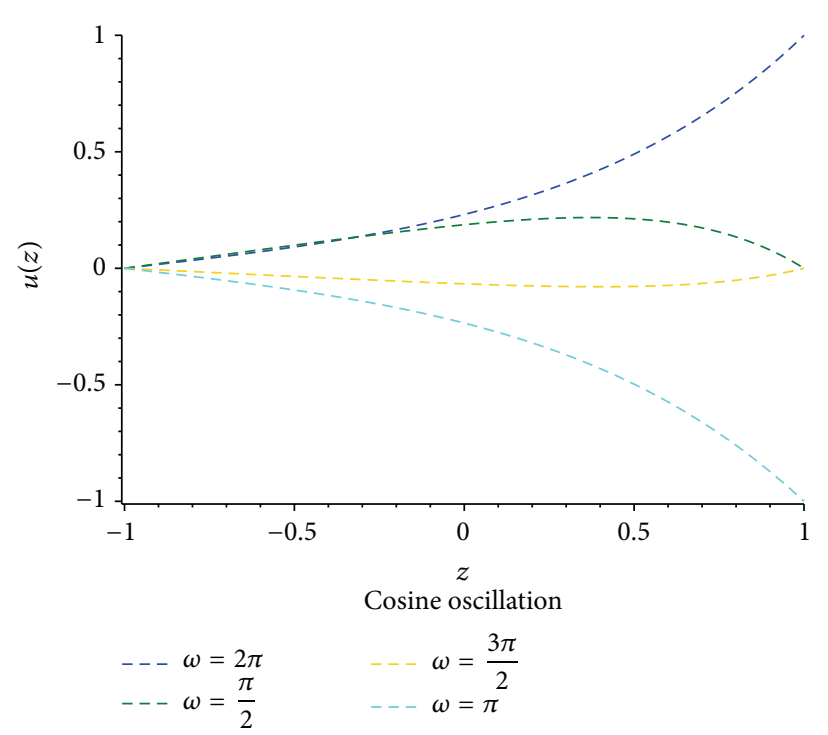

(a)

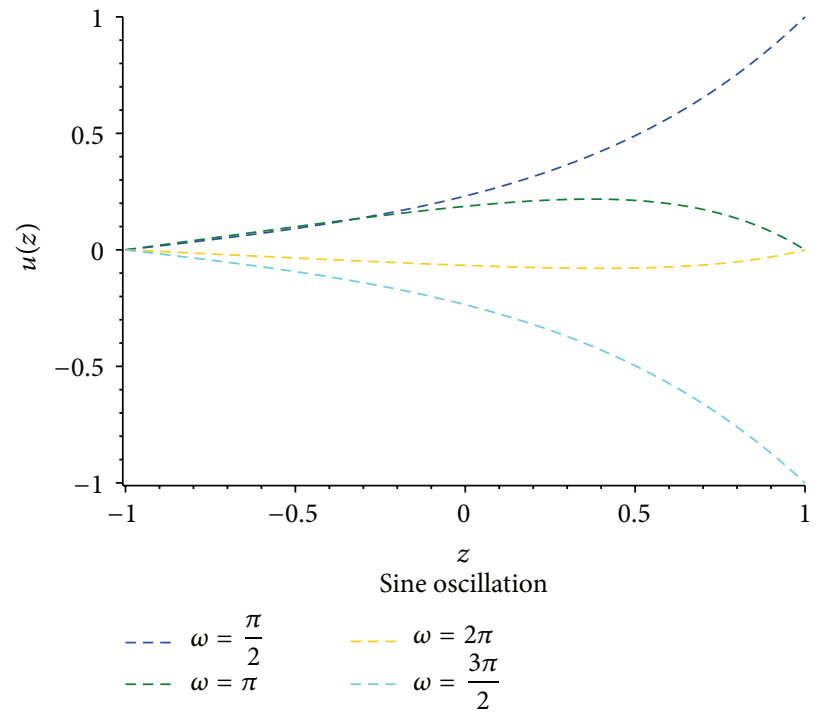

(b)

FIgURE 3: Plot of velocity field $u$ for varying values of $\omega$ for small time $t=1$ and $\alpha=0.5, \xi=-0.7$, and $\beta=0.5$. 


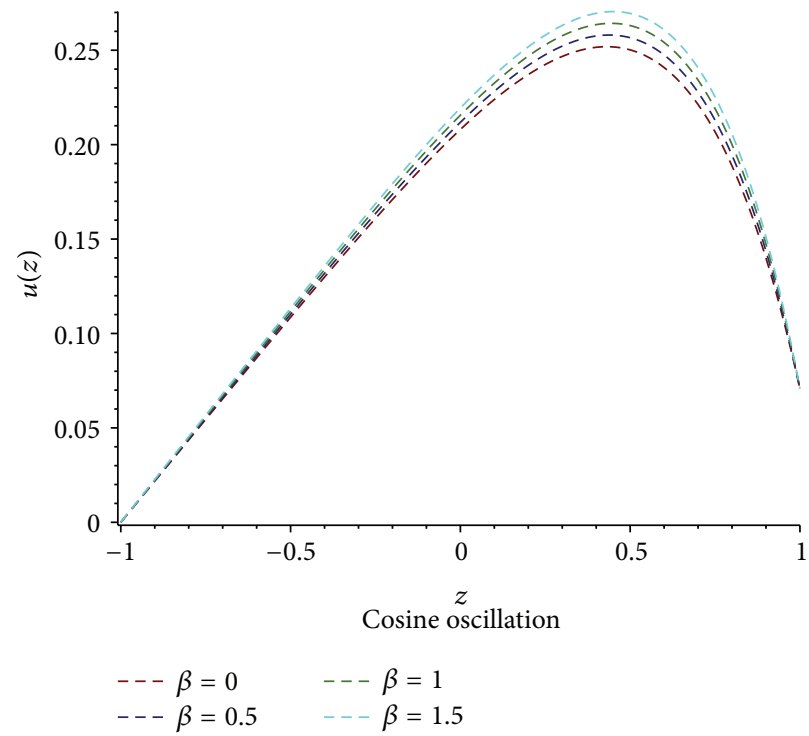

(a)

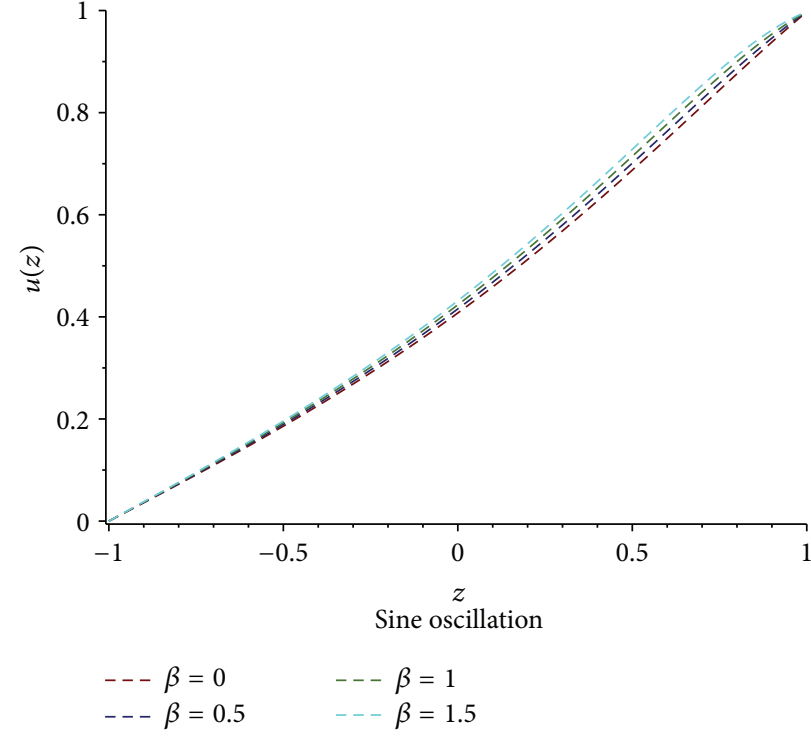

(b)

FIGURE 4: Plot of velocity field $u$ for varying values of $\beta$ for small value of time $t=1$ and $\alpha=0.5$, and $\xi=-0.7$.

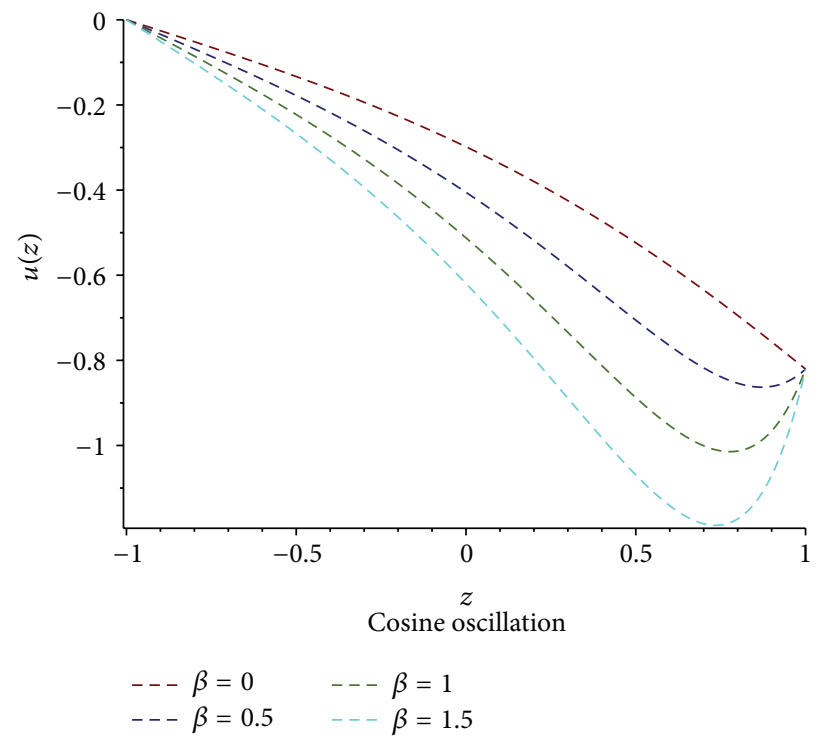

(a)

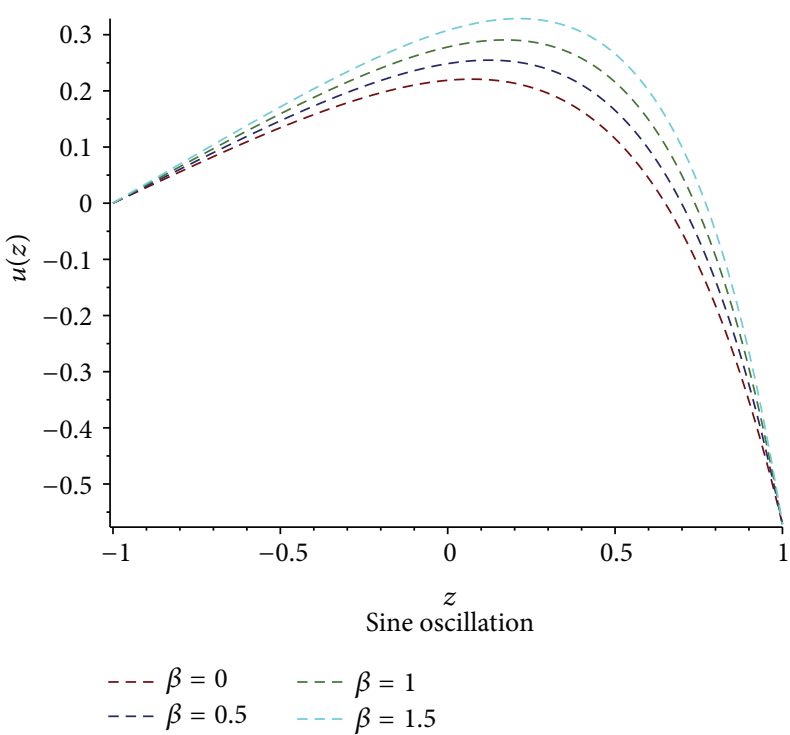

(b)

Figure 5: Plot of velocity field $u$ for varying values of $\beta$ for large value of time $t=2.5$ and $\alpha=0.5$, and $\xi=-0.7$.

In Figure 6 the wall stress $\left(\tau_{\omega}=\partial u(t, z) / \partial z\right)$ is plotted against the dimensionless space coordinate $z$. For cosine oscillations, it is observed that the wall stress increases near the lower plate, whereas it decreases near the upper plate. This is because of the simultaneous suction and blowing phenomena at the lower plate and upper plate, respectively. The effects get reversed in the case of sine oscillations.

\section{Conclusion Remark}

In this paper, a new technique is proposed to obtain analytical solutions of the transient flow of viscoelastic third grade fluid. We applied a new technique to our problem, which is also solved using HPM and OHAM. We obtained an explicit analytic solution of the 2D laminar transient third grade 


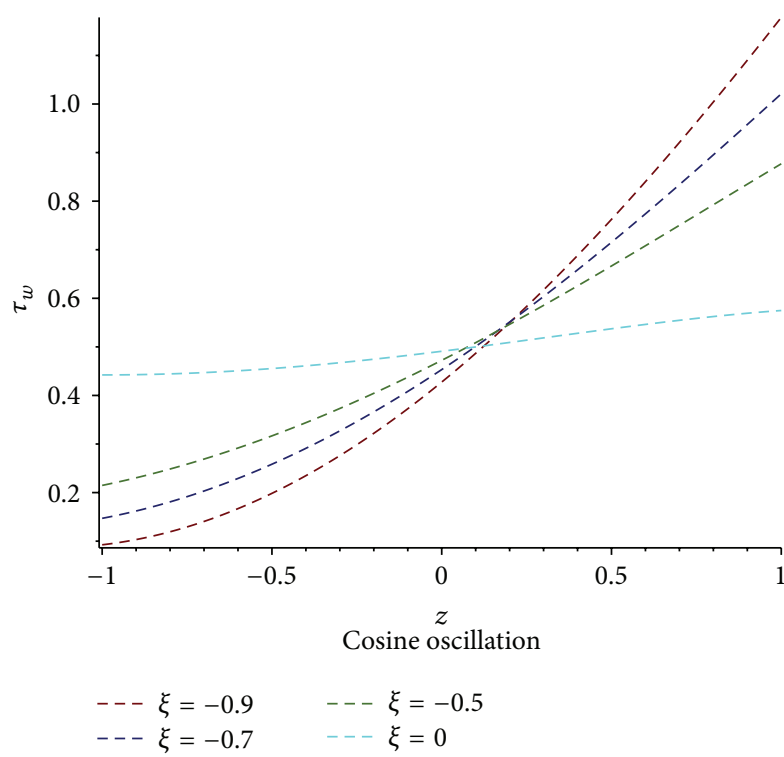

(a)

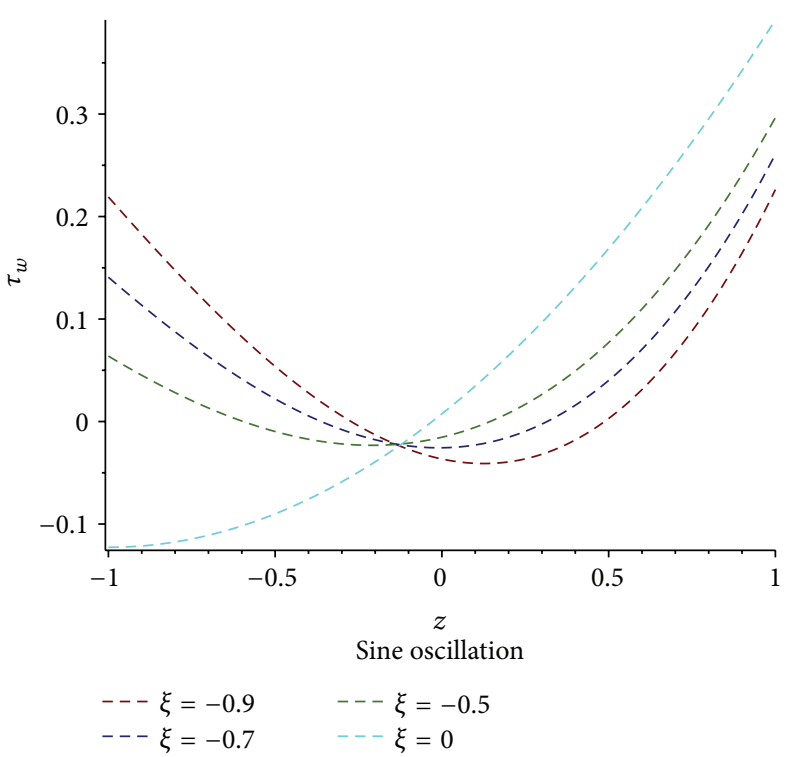

(b)

FIGURE 6: Effect of blowing on wall stress $\tau_{\omega}$ when $\omega=0.5, \alpha=0.5, t=0.2$, and $\beta=0.5$ are fixed.

flow over a vertical channel with oscillation motions on a transpiration wall. This explicit analytic solution is valid in the whole transient region $0<t<1.5$. The results obtained using HPM and OHAM are compared with the solutions of the new technique. We observed that we get accurate results using new method, by comparing with OHAM results.

The result has shown that the influence of the third grade parameter $\beta$ on the fluid motion is significant only for small values of time $t$.

This approach seems to be useful and can be used to obtain other analytical solutions for other moving boundary layer transient equations in fluid mechanics Fan et al. [11] and Noghrehabadi et al. [12].

\section{Conflict of Interests}

The authors declare that there is no conflict of interests regarding the publication of this paper.

\section{Authors' Contribution}

Associate Professor Dr. Rozaini Roslan and Dr. Mahathir Bin Mohamad have made significant contributions in the revised version of the paper. They assisted immensely in the comparison between HPM and OHAM. Their contributions have helped in making positive change in the paper.

\section{References}

[1] K. Fakhar, Z. Xu, and C. Yi, "Exact solutions of a third grade fluid flow on a porous plate," Applied Mathematics and Computation, vol. 202, no. 1, pp. 376-382, 2008.
[2] M. Danish and S. Kumar, "Exact analytical solutions for the Poiseuille and Couette-Poiseuille flow of third grade fluid between parallel plates," Communications in Nonlinear Science and Numerical Simulation, vol. 17, no. 3, pp. 1089-1097, 2012.

[3] M. Abdulhameed, I. Khan, D. Vieru, and S. Shafie, "Exact solutions for unsteady flow of a second grade fluid generated by an oscillating wall with transpiration," Applied Mathematics and Mechanics, vol. 35, no. 7, pp. 821-830, 2014.

[4] M. Ayub, A. Rasheed, and T. Hayat, "Exact flow of a third grade fluid past a porous plate using homotopy analysis method," International Journal of Engineering Science, vol. 41, no. 18, pp. 2091-2103, 2003.

[5] J. He, "Application of homotopy perturbation method to nonlinear wave equations," Chaos, Solitons and Fractals, vol. 26, no. 3, pp. 695-700, 2005.

[6] A. Ghorbani, "Beyond Adomian polynomials: he polynomials," Chaos, Solitons and Fractals, vol. 39, no. 3, pp. 1486-1492, 2009.

[7] Y. Khan and Q. Wu, "Homotopy perturbation transform method for nonlinear equations using He's polynomials," Computers \& Mathematics with Applications, vol. 61, no. 8, pp. 19631967, 2011.

[8] Y. Khan and Z. Smarda, "A novel computing approach for third order boundary layer equation," Sains Malaysiana, vol. 41, no. 11, pp. 1489-1493, 2012.

[9] A. Nazari-Golshan, S. S. Nourazar, H. Ghafoori-Fard, A. Yildirim, and A. Campo, "A modified homotopy perturbation method coupled with the Fourier transform for nonlinear and singular Lane-Emden equations," Applied Mathematics Letters, vol. 26, no. 10, pp. 1018-1025, 2013.

[10] V. Marinca and N. Herișanu, "Application of optimal homotopy asymptotic method for solving nonlinear equations arising in heat transfer," International Communications in Heat and Mass Transfer, vol. 35, no. 6, pp. 710-715, 2008. 
[11] T. Fan, H. Xu, and I. Pop, "Mixed convection heat transfer in horizontal channel filled with nanofluids," Applied Mathematics and Mechanics, vol. 34, no. 3, pp. 339-350, 2013.

[12] A. Noghrehabadi, A. Behseresht, and M. Ghalambaz, "Natural convection of nanofluid over vertical plate embedded in porous medium: prescribed surface heat flux," Applied Mathematics and Mechanics, vol. 34, no. 6, pp. 669-686, 2013. 

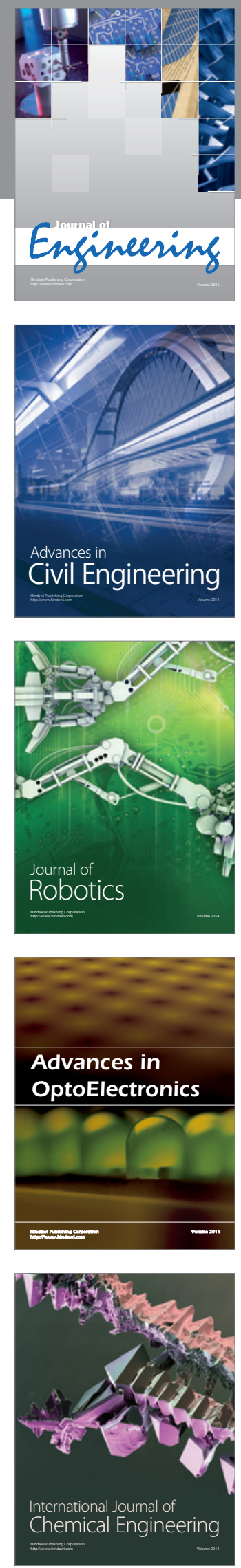

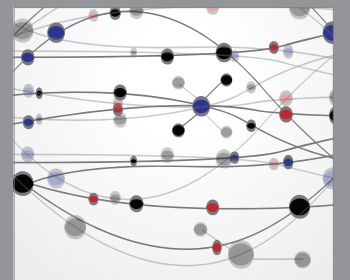

The Scientific World Journal
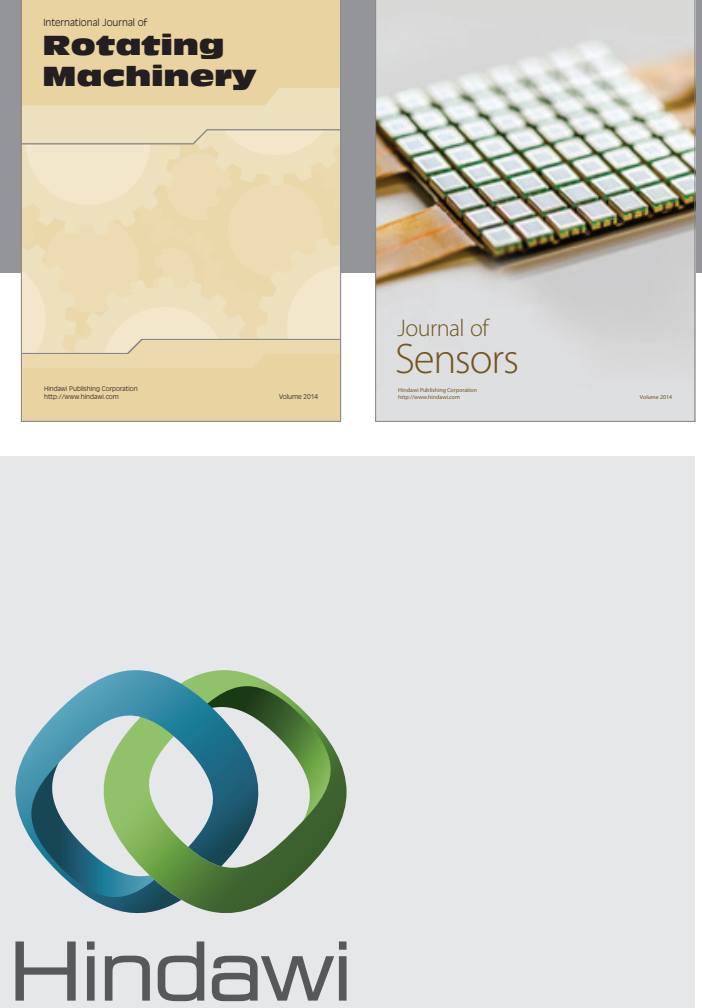

Submit your manuscripts at http://www.hindawi.com
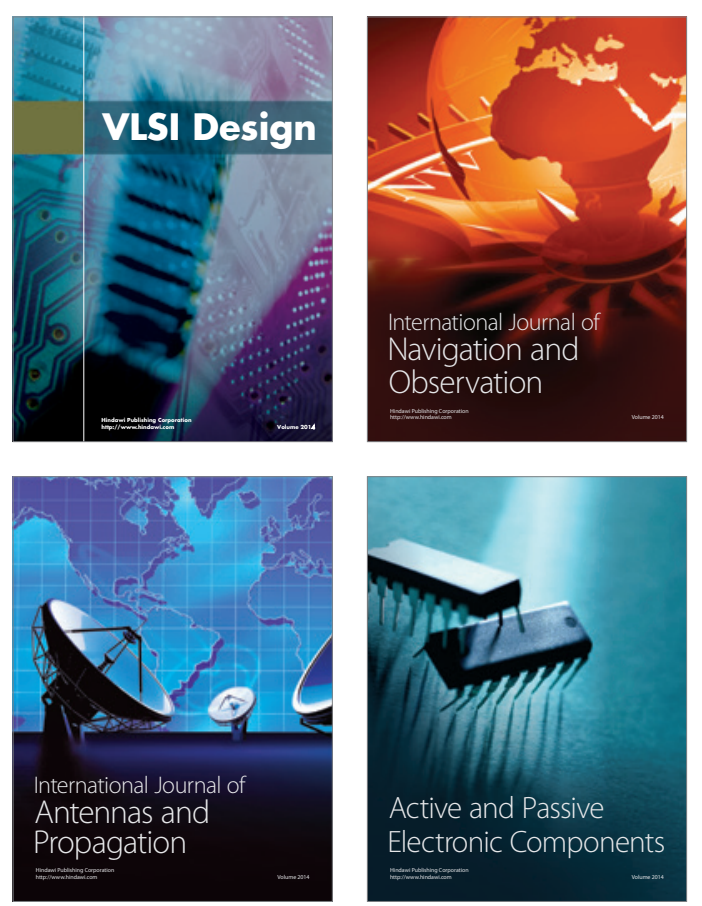
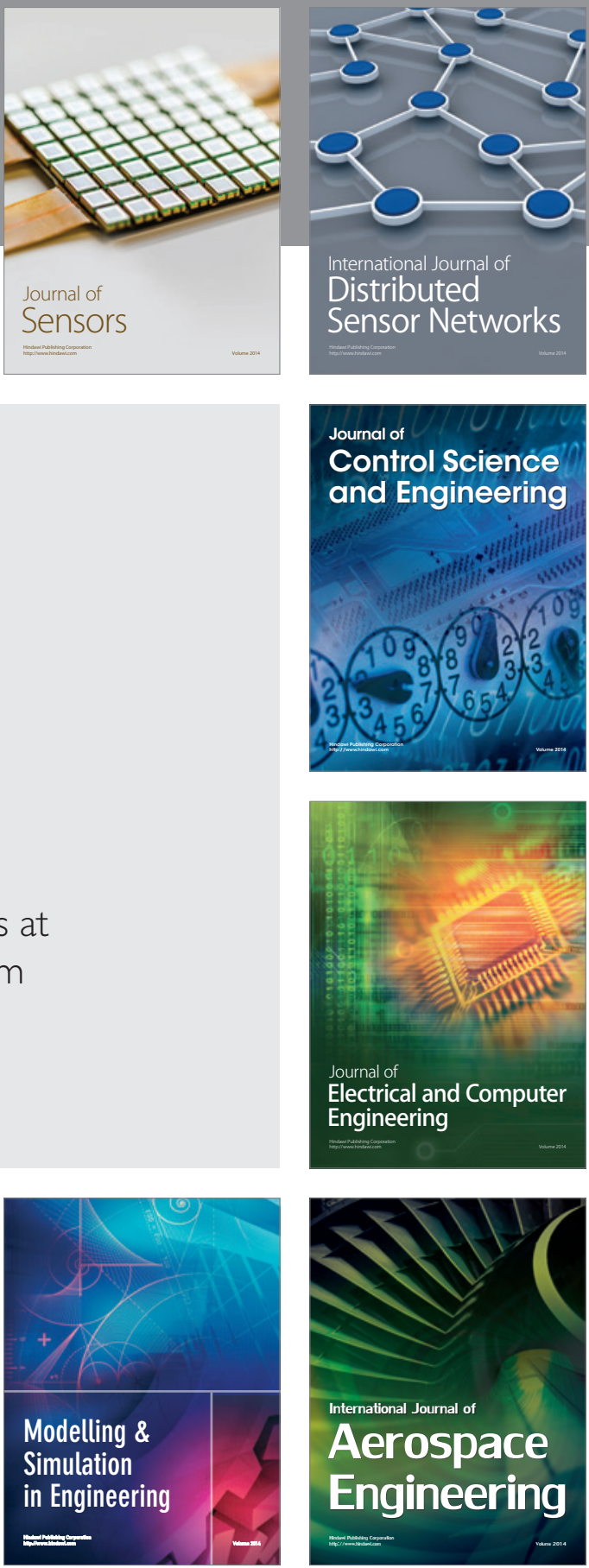

Journal of

Control Science

and Engineering
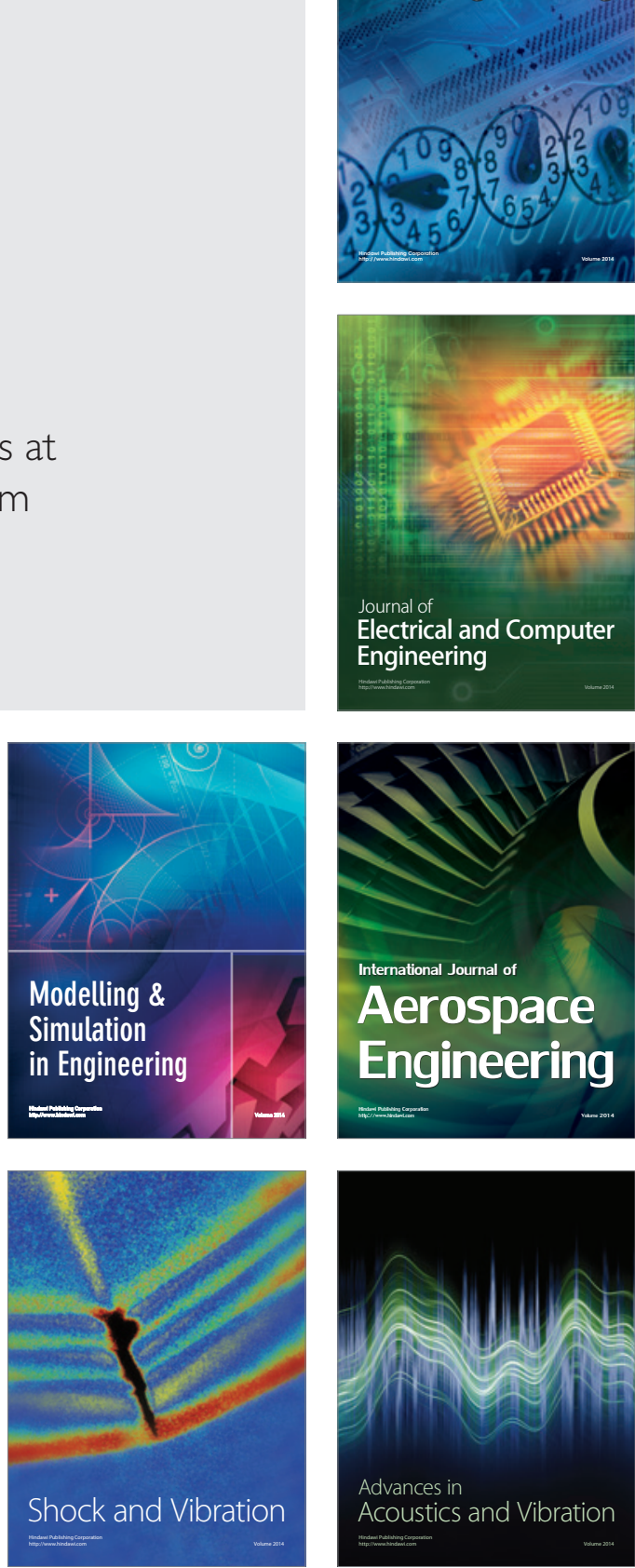